\title{
RESEARCH ON THE CONSTRUCTION OF ZHAOQING URBAN ECOLOGICAL INFRASTRUCTURE BASED ON ECOLOGICAL SECURITY
}

\author{
ZHANG, $\mathrm{H}$. \\ College of Life Sciences, Zhaoqing University, Guangdong, China \\ (e-mail: zhang-hongg@163.com) \\ (Received 19 $9^{\text {th }}$ Mar 2019; accepted 22 $2^{\text {nd }}$ May 2019)
}

\begin{abstract}
In the process of urbanization, the region faces environmental problems such as destruction of ecological corridors, fragmentation of biological habitats, and soil erosion, which pose potential risks to the regional ecological environment. Therefore, it is of great significance to identify lands with ecological conflict, establish core ecological protection zones and ecological corridors, and build an ecological security pattern for regional sustainable development. The research subject of this paper is Zhaoqing area. From the perspective of landscape ecological security pattern, it identifies the green land in Zhaoqing City, hydrological system, local culture, natural ecological protection, and the chance of geological disasters, and combines RS (Remote Sensing) and GIS (Geographic Information System) technology to evaluate green land and identify environmental core protection. Urban ecological infrastructure patterns are built in district, ecological corridors and ecological buffer zones and land with ecological conflict is curbed form expansion. The study found that it is necessary to carry out controlled development of ecological conflict zones such as the Xijiang River Basin, the Beiling Mountain Forest Land, and the Gaoyao Plain to ensure the safety of the fundamental ecological facilities in Zhaoqing.
\end{abstract}

Keywords: core ecological protection zone, ecological assessment, ecological source, ecological buffer zone, control development

\section{Introduction}

At present, China's rapid economic development promotes urban construction, but it is accompanied by an ecological environmental crisis that threatens the national ecological security. The expansion rate of urban construction land in China is significantly higher than the population growth rate. In the past 34 years, China's urban construction land has increased by 6.44 times, with an average annual growth rate of $6.27 \%$. The per capita construction area of Chinese cities is $129.57 \mathrm{~m}^{2}$, which significantly exceeds the national standards, and is also significantly higher than the average of $84.4 \mathrm{~m}^{2}$ per capita in developed countries and $83.3 \mathrm{~m}^{2}$ per capita in other developing countries (The 13th Five-Year Ecological Protection Plan of the Ministry of Environmental Protection of China, 2016). This trend extends from the eastern coastal areas to the inland, and from the central towns rural areas, forming a situation of "city in green retreat", destroying the original ecological balance, causing regional ecological, environmental crisis and seriously affecting regional ecological security. For the sustainable development of the city, the fundamental green land of the city must be protected and the urban ecosystem must be perfected. Protection of green land is the most critical task at the moment. To improve the urban ecosystem, it is necessary to discriminate the urban environmental protection area, rationally plan the urban spatial structure and urban development direction, optimize the urban functional structure, and reduce the contradiction between urban expansion and ecological land use.

In order to solve the contradiction between urban expansion and ecological land use, Chinese and foreign scholars have made a series of explorations and achieved specific 
results. For example, the savvy growth theory proposed by American scholar Doyle focuses on the balance between urban expansion and ecological protection. The ecological planning theory put forward by domestic scholar Yu Kongjian and others put emphasis on the priority of green land protection and the control of the integrity of the ecological structure. In recent years, Chen Yimin and Peng Jiajie have used the landscape ecological index and landscape ecological risk assessment methods to construct a spatial conflict measurement model to assess the spatial conflict level under rapid urbanization. These theories emphasize the balanced relationship between environmental protection and urban development and have been widely recognized by the urban planning industry. Based on the GIS spatial analysis model, this paper simulates and analyzes the spatial conflicts and ecological security risks of urban ecological land in Zhaoqing City, and provides a scientific basis for urban development and protection.

\section{Ecological infrastructure concept}

The concept of ecological infrastructure was first proposed in the 1984 UNESCO Man and Biosphere Programme (MAB). The ecological infrastructure is a natural system, which is essential for the survival of urban and rural residents and their inhabitants. It provides residents with fresh air, food, sports, recreation, safe haven, aesthetics and education. With the deepening of research, the idea of ecological infrastructure is reflected in the emphasis from simple "protection" to the use of EI (ecological infrastructure) to guide the development of the city, thus achieving the "EIoriented urban development" approach (Yu et al., 2005).

In the process of urbanization in China, we face the problem of urbanization and ecological environmental protection. Unreasonable urban development and construction have damaged the regional ecosystem structure, resulting in weakened ecosystem service functions, seriously threatening residents' production and life, and affecting sustainable environmental development. It is crucial for the government, especially urban planning administrators, to find out methods that benefit the balance between urban development pressure and ecological environment protection, the restoration of the natural system of the city, and sustainable urban development. Urban safety development depends on the basic maintenance of ecosystem services.

\section{Landscape ecological security pattern theory}

There are some potential spatial patterns in urban landscapes, which are composed of vital ecological nodes, corridors and location relationships. This pattern plays a crucial role in maintaining and controlling an ecological process called landscape ecology ( $\mathrm{Yu}$, 1999). Safety pattern: Landscape ecological security pattern is based on landscape ecology theory and method in the late 1990s. Based on the relationship between landscape processes and patterns, it is critical to distinguish the health and safety of these processes through the analysis and simulation of landscape processes. Landscape pattern: The landscape ecological security approach takes the landscape process (including regional hydrological cycle, species space movement, disaster process diffusion and urban sprawl expansion) as a process of achieving landscape control and coverage by overcoming spatial resistance. To achieve effective control and coverage, strategically important landscapes must be captured. Elements, spatial locations, and 
connections: The pattern formed by such vital elements, strategic locations and connections is the landscape ecological security pattern, which is essential for maintaining and controlling ecological processes or other horizontal processes ( $\mathrm{Yu}$, 1999).

At present, the relatively mature method for identifying the landscape ecological security pattern is to realize landscape control by constructing the Minimum Cumulative Resistance (MCR) of the ecological process. The MCR model is based on the GIS spatial analysis module to analyze the degree of separation of landscape patches and serve as the basis for landscape pattern optimization. The landscape pattern optimization MCR model is mainly realized in the GIS by relying on the minimum cost distance (Cost Distance) model. By using the landscape resistance surface characteristics to distinguish the landscape ecological strategic points, it extends to the discrimination of the landscape ecological security pattern. The equation for calculating the MCR model can be expressed as follows:

$$
M C R=\sum_{j=n}^{i=m}\left(D_{j i} \times R_{i}\right)
$$

where $f$ is an unknown positive function, reflecting the positive correlation between the minimum resistance of any point in space and the spatial distance of the base $i$ of a landscape that it traverses and the characteristics of the landscape base. $D_{i j}$ is the spatial distance of the base $i$ of a landscape from the source $j$ to a point in space; $R_{i}$ is the resistance of landscape $i$ to the movement of a species. Although the function $f$ is unknown, the $\left(D_{i j} \times R_{i}\right)$ value can be regarded as a relative measure of the path of a species from a source to a point in space, where the maximum value of resistance from all sources to that point is used. Measuring the accessibility of this point, the constructed resistance surface can reflect the potential possibilities and trends of species movement (Ahern, 1995; Yu, 1996). The landscape ecological security pattern theory as a dynamic spatial structure model is a method of constructing ecological infrastructure. It regards the horizontal landscape process as a process of overcoming spatial resistance to achieve landscape control and coverage, and requires corresponding constraints and discriminant parameters, theoretical and technical support in hydrology and ecology.

\section{Construction of ecological infrastructure in Zhaoqing urban area}

By analyzing ecological services and ecological process functions, American landscape ecology experts Ahern and Ndubisi categorized ecological infrastructure into three types: Biotic, Abiotic, and Culture (Ndubisi, 2002). This comprehensive and inclusive model based on landscape ecology recognizes the interaction between biotic and abiotic systems and humans (Gan et al., 2007). It is also known as the ABC model and is widely used in the planning and construction of ecological infrastructure in the application in United States (Xiao et al., 2017). As a result of ecological security pattern of different types of landscapes, ecological infrastructure reflects the realization of ecological functions and the integrity of ecological processes. The completion of ecological processes requires the support of ecological space, and the ecological infrastructure is the guarantee of ecological functions.

This paper analyzes the non-construction land structure of Zhaoqing City, draws on the ecological infrastructure concept, and uses the $\mathrm{ABC}$ model as a framework to 
identify the landscape ecological security pattern. Through the computational composition and spatial superposition of the Zhaoqing urban ecological infrastructure network, the geological, biological, hydrological and the landscape ecological security pattern of local culture forms the ecological infrastructure for the healthy development of Zhaoqing.

\section{Research scope and data source}

Zhaoqing City is located in the central and western part of Guangdong Province, northwest of the Pearl River Delta. The city has jurisdiction over Dinghu District, Duanzhou District, Gaoyao District, Zhaoqing High-tech Zone (Dawang), Zhaoqing New District, and other five districts. The total area of the city is $2990 \mathrm{~km}^{2}$. The overall planning of Zhaoqing City is characterized by high terrain in the northwest and south, and flat terrain in the northeast to southwest. The low-mountain landforms in the northwest and the south are mainly distributed in the main mountain bodies such as Beiling Mountain, Dinghu Mountain and Rouge Mountain. The main urban construction land is distributed in the northeast to southwest plain. The basic pattern of Zhaoqing City from south to north is "river, city, lake, mountain, forest", the spatial pattern of the strip city, and the city forms a trend of developing eastward with the end state as the center. With the difficulty and timing of data acquisition being considered, this investigation selects the remote sensing image of LANDSAT (Tursing Landsat System) TM series as the main data source, and combines Zhaoqing urban topographic map, land use status map and land use change table, processing and analysis.

\section{Zhaoqing City hydrological system security pattern}

Floods are inevitable as part of the river's natural processes, but floods can be minimized through ecological planning. From the perspective of spatial planning, by studying the distribution pattern of floodplain, provides a basis for planning land use decision-making, and planning flood-prone areas as wetlands and open spaces is an effective way to reduce flood disasters and their effects (Xiao et al., 2017). The flood safety pattern of Zhaoqing City should be started from different scales of watersheds. By establishing a coordinated flood control system, corresponding flood control, measures should be in accordance with the risk level.

Since this investigation is based on GIS simulations, it is necessary to calculate the flood level of different safety levels or the adjustable flood flow and the flow that can be safely discharged and the adjustable flood storage. Therefore, it is necessary to obtain historical hydrological and monitored storm runoff data to calculate the flood level. The reasonable height and the area where the flood spreads is reduced. At the same time, by collecting the flooding range of historic floods, the different ranges of floodplain, flood detention zone and flood protection zone are analyzed. Moreover, the scale, pattern and river channel buffer width of different flood risks are determined, and the star lake and east channel are established. Different levels of safety concerning Honghu, Poyang Lake and other ecological wetlands, rivers and rivers coordinated flood control dynamic system are determined according to different risk levels.

The hydrological analysis module in GIS is a means to effectively analyze and simulate hydrological processes, which can simulate the regional runoff process and identify potential wetlands. The hydrological analysis module generally requires digital elevation data (DEM) to simulate the runoff process, and then establishes a flood 
surface based on the flood level at different safety levels to obtain the flood submerged range. The Zhaoqing City is mainly composed of the Xijiang River Basin and the Xinghu Wetland, the Jiukeng River Reservoir (Kowloon Lake) Wetland Core Area and the New Area Wetland Core Area. The hydrological analysis module in GIS comprehensively evaluates the ecological and social benefits of the Xijiang River Basin and its wetlands, and follows the protection of its ecosystem health. The spatial and spatial pattern of wetland resources is planned for the connectivity between the corridors and wetlands, the stability of large wetland patches, and the ecological channels provided for urban groups.

It is selected as a concrete case to discuss the flood control safety pattern based on flood volume. Firstly, the CAD contour data of Zhaoqing New District is converted into DEM data as the basic data of runoff process simulation. Through GIS simulation of the runoff process, the location and extent of potential wetlands can be obtained by encountering stagnation points in low-lying areas, mainly distributed in the wet areas of the Xijiang River Basin. Based on the Arc GIS, the flood surface elevation raster data is established based on the flood water level, and the simulated water level of each point in the area is obtained (Yu, 1999). Subsequently, we calculate the difference between the elevation of the flood surface and the terrain elevation to obtain the flood inundation range of different return periods. The main flood control area in Zhaoqing is along the Xijiang River and some rivers (Fig. 1).

\section{Zhaoqing City geological hazard safety pattern}

Through the sensitivity analysis of regional geological disasters in Zhaoqing City, we summarized the distribution of geological disaster safety patterns. First, we determined the source of geological hazards by superimposing the spatial distribution of various geological hazards such as mountain collapse, landslide, slip, ground subsidence and ground fissure on the Zhaoqing regional GIS map. Subsequently, through the analysis of the types of local disasters and the analysis of land use patterns in disaster-prone areas, the regional and spatial linkages that play a vital role in the protection of geological disasters were determined. The extent of the buffer zone is affected by factors such as the type of geological hazard, development intensity, distribution, the frequency of occurrence, topographic and geological conditions, precipitation conditions and intensity of human activities (Russell and Wang, 1997). Finally, based on the spatial superposition of the spatial distribution of various disaster sources, the safety pattern of geological disasters with different safety levels was obtained.

The geological disaster factors of Zhaoqing City mainly include various geological disaster factors such as mountain collapse, landslide, slippage, land subsidence and ground fissure. Based on GIS, the elevation and slope are graded to obtain the sensitivity distribution of geological disasters. The spatial distribution of geological disaster sources is identified by superimposing various geological disaster factors (Table 1; Fig. 2).

\section{Zhaoqing City biosafety security pattern}

The biosafety safety pattern is mainly to construct the minimum resistance model (Eq. 1) to simulate the minimum resistance value that the species needs to overcome in space motion, and then to identify the landscape safety pattern by analyzing the resistance surface outside the core habitat (source). The safe pattern of biological processes 
generally consists of sources (core habitats), buffer zones (low-resistance areas around the source), corridors and radiant channels (low-resistance channels connecting multiple sources), and strategic points (critical to biological processes), which constitute elements of the landscape (Yu et al., 2005). This method of identifying the landscape safety pattern can select the model to analyze, so as to achieve the identification of the "matrix-corridorplaque" model in landscape ecology. It is best to select migratory birds as indicator species, which is conducive to the communication between elements. Sexuality reflects the strategy of the overall biological safety of the region.

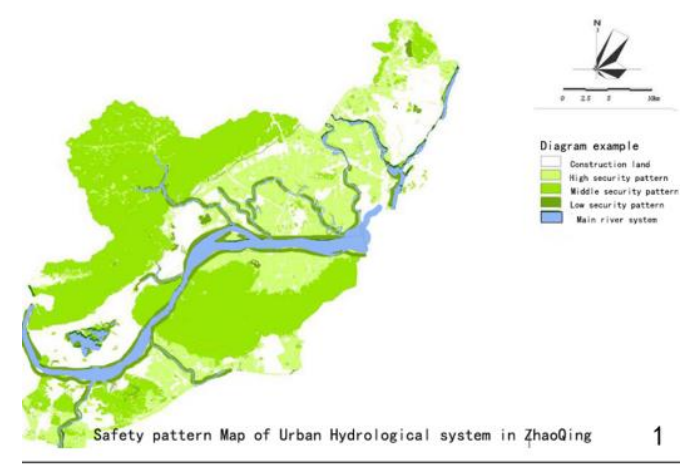

Figure 1. Zhaoqing urban hydrological security map

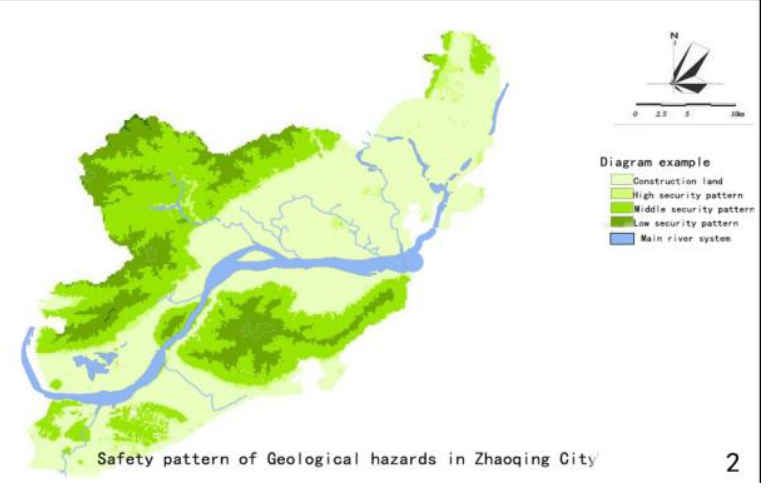

Figure 2. Zhaoqing urban geological security map

Table 1. Identification of geological disaster sources in Zhaoqing City

\begin{tabular}{c|c|c|c}
\hline $\begin{array}{c}\text { Level of safety } \\
\text { Risk source }\end{array}$ & $\begin{array}{c}\text { Debris flow, landslide, } \\
\text { collapse }\end{array}$ & $\begin{array}{c}\text { Fracture } \\
\text { zone }\end{array}$ & Ground setting \\
\hline Low safety level & $\begin{array}{c}\text { Elevation }>1000 \mathrm{~m}, \\
\text { slope }>30 \text { degree, collapse } \\
\text { center }\end{array}$ & $\begin{array}{c}100 \mathrm{~m} \text { around } \\
\text { the fault zone }\end{array}$ & $\begin{array}{c}\text { Surface subsidence center zone, } \\
\text { cumulative settlement }>2.0 \mathrm{~m}\end{array}$ \\
Medium safety level & $\begin{array}{c}\text { The height is } 500 \mathrm{~m}, \text { the } \\
\text { slope is } 15-30^{\circ}, \text { the center } \\
\text { of collapse is } 300 \mathrm{~m}\end{array}$ & $\begin{array}{c}300 \mathrm{~m} \text { around } \\
\text { the fault zone }\end{array}$ & $\begin{array}{c}150 \mathrm{~m} \text { around the land subsidence } \\
\text { center, cumulative settlement }>1.5 \mathrm{~m}\end{array}$ \\
\hline High safety level & Collapse center $500 \mathrm{~m}$ & $\begin{array}{c}500 \mathrm{~m} \text { around } \\
\text { the fracture } \\
\text { zone }\end{array}$ & $\begin{array}{c}\text { The circumference of the center of } \\
\text { the heart is } 300 \mathrm{~m}, \text { and the cumulative } \\
\text { subsidence is } 0.3 \mathrm{cu} 1.0 \mathrm{~m}\end{array}$ \\
\hline
\end{tabular}


Wetland and forest land with an area larger than $25 \mathrm{hm}^{2}$ in the Zhaoqing City area are selected as the habitat of protected species. The spatial resistance coefficient of different land cover types is set by using the spatial resistance coefficient of different land cover types (Table 2). Superimposed, the resistance surface of the space movement of the protected species and the ecological corridor with the least space resistance of the species movement are obtained, and the bio-protection safety pattern of Zhaoqing City based on the analysis of the horizontal ecological process is formed. According to the calculation, the three ecological green nucleuses of Dinghushan, Rougekeshan and Sihui12 are formed. The three ecological corridors of Xijiang River, Minjiang River and Beijiang River are beneficial to the protection of biodiversity (Fig. 3).

Table 2. Spatial resistance coefficient of minimum cost distance model analysis

\begin{tabular}{c|c|c|c}
\hline Attributes & $\begin{array}{c}\text { Coefficient of } \\
\text { drag }\end{array}$ & Attributes & $\begin{array}{c}\text { Coefficient of } \\
\text { drag }\end{array}$ \\
\hline Water system river & 150 & High density construction land & 10000 \\
Forest land & 275 & Highway & 600 \\
Open forest land & 50 & Tidal flat & 150 \\
Shrub forest & 40 & Natural conservation land & -5 \\
Base pond & 100 & Farmland & 325 \\
Meadow & 30 & & \\
Dry farm & 300 & & \\
Low density construction land & 1000 & & \\
\hline
\end{tabular}

\section{Zhaoqing City's local cultural security pattern}

It is necessary to study the current landscape from the historical perspective as a result of past natural processes and human interference (Costanza, 2008). From the perspective of ecosystem service functions, it is critical to integrate the natural environment characteristics and local cultural characteristics into the local landscape and cultural heritage resources. They have many functions such as cultural diversity, spiritual and religious values, educational values, social connections, aesthetics and enlightenment, cultural heritage values, recreation and ecotourism. The introduction of the concept of ecosystem service function makes people's understanding of the local cultural landscape not only stay in the cultural sense, but the dual carrier of culture and natural function (Jongman, 1995).

The study of the local cultural landscape safety pattern is the "source" of the local cultural landscape elements in the region, and regards people's recreation activities as a process of horizontal expansion. Through the establishment of spatial resistance surface, the safety pattern of the local cultural landscape is constructed. As the birthplace of Guangfu culture, Zhaoqing selects the cultural landscape protection units such as the Zhaoqing Fucheng historical and cultural district and the typical Guangfu village and the Gaojiu area, the Sangji fish pond agricultural culture demonstration area as the source of the local cultural security pattern. The land cover type sets different spatial resistance coefficients, and the minimum cumulative resistance surface is established by the distance model to obtain the safety landscape of the local culture landscape with different safety levels (Fig. 4). 


\section{Zhaoqing comprehensive ecological infrastructure}

Based on the above-mentioned hydrological system safety pattern, geological disaster safety pattern, biological protection safety pattern and local cultural security pattern, spatial computing and superposition based on GIS, and the comprehensive ecological infrastructure of Zhaoqing City with different security levels are determined (Fig. 3; Table 3).

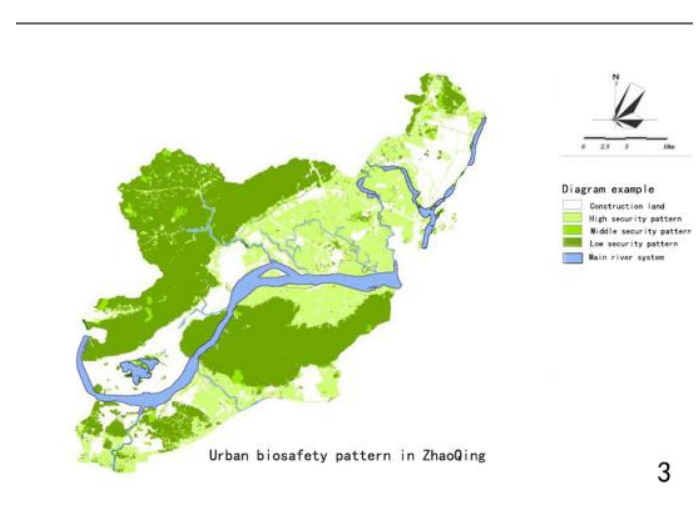

Figure 3. Zhaoqing City biosafety map

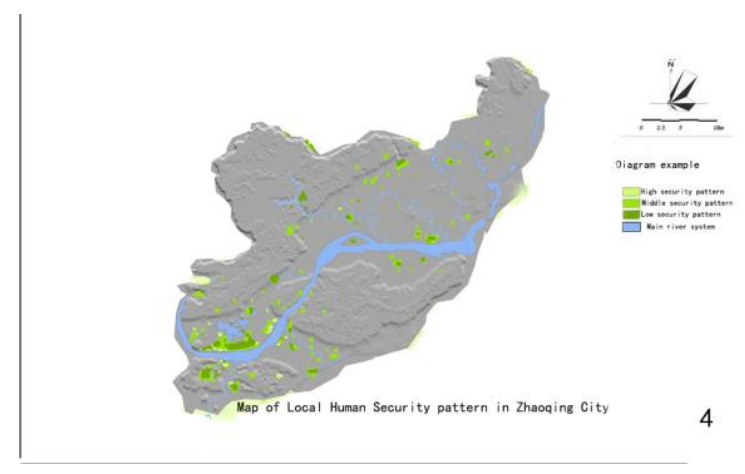

Figure 4. Zhaoqing City humanities security map

Table 3. Area and proportion of ecological infrastructure at different safety levels in Zhaoqing City

\begin{tabular}{c|c|c|c|c}
\hline \multirow{2}{*}{ Ecological security level } & \multicolumn{2}{|c|}{ Ecological infrastructure occupation } & \multicolumn{2}{c}{ Construction land } \\
\cline { 2 - 5 } & Area/km $\mathbf{k m}^{\mathbf{2}}$ & Scale/\% & Area/km & Scale/\% \\
\hline Low safety level & 1072 & 35.86 & 1918 & 64.14 \\
Medium safety level & 1829 & 61.18 & 1161 & 38.82 \\
High safety level & 2321 & 77.64 & 669 & 22.36 \\
\hline
\end{tabular}

The comprehensive ecological infrastructure of Zhaoqing City is divided into three different security levels: EI with high security level is the ideal state to realize the functions of various ecosystems in the region. The security scope can be moderately opened according to local conditions. The medium-safety level EI is an appropriate scale to maintain a complete ecosystem of the region, and can be constructed within 
certain limits. The low-safety level EI is the threshold for ensuring urban ecological security, and is an insurmountable red line in urban and rural development and construction. Zhaoqing's current development is at a high level of safety, but it is still prudent to develop in some highly ecologically sensitive areas.

\section{Zhaoqing City ecological conflict space recognition}

The identification of the ecological conflict space in Zhaoqing City depends on the map layer map superposition method. By superimposing the GIS map of the medium security level in Zhaoqing City on the spatial distribution map of the current construction land, it can be found that the ecological conflict space of Zhaoqing City is concentrated in the edge belt of the existing built-up area, such as the Beiling High Ridge Area of Zhaoqing and Zhaoqing High-tech Zone (large Wang) in the north and the periphery of Dinghu Mountain in Zhaoqing New District. In addition, the ecological infrastructure of the low (bottom line) safety level in Duanzhou District and Dinghu District is affected by the embedded construction land, and the ecological conflict area is distributed along the mainstream of the Xijiang River, which has a great impact on the ecological environment. The ecological conflict area in Duanzhou District is dotted, although the area is small, because it is the red line of regional ecological security, the impact on regional ecological processes and ecological functions is more prominent (Fig. 5). Thus, it should be controlled. In addition, it is necessary to control development in the vicinity of Jiukenghe Reservoir and Dinghu Mountain in ecologically sensitive areas should be controlled for development.

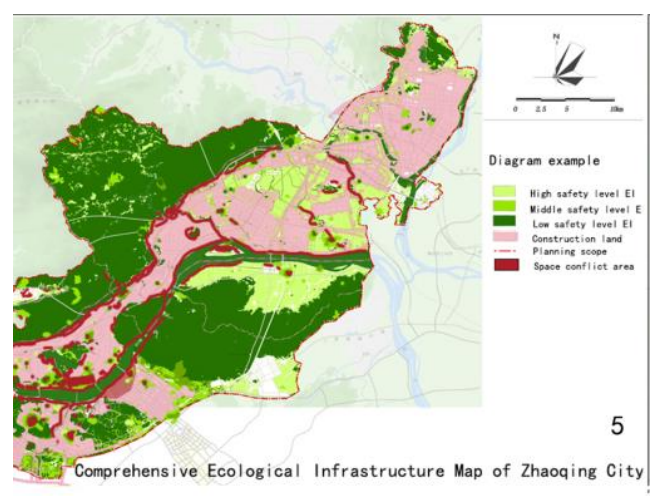

Figure 5. Zhaoqing City ecological conflict zone map

\section{Conclusions}

With Zhaoqing urban area as the research object, this investigation quantitatively evaluates hydrological systems, biological protection and local culture services, and the chance of geological disasters. From the perspective of natural land evolution, the investigation is based on landscape ecological security pattern theory and MCR model, utilizing RS and GIS space. With the aid of analytical techniques, through the spatialization of spatial data and spatial model calculations, the non-biologicalbiological-cultural analysis framework was used to identify the geological disasters, hydrological systems, biological protection and local cultural security patterns in Zhaoqing City. Superimposing a single ecological security pattern to achieve an 
integrated ecological infrastructure with different levels of security (Fig. 6). The results show that ecological land covers an area of $657.8 \mathrm{~km}^{2}$, accounting for $22 \%$ of the research land, of which the ecological land with an area larger than $10 \mathrm{~km}^{2}$ is used as the source, about $568.1 \mathrm{~km}^{2}$, accounting for $19 \%$ of the research land, mainly the North Ridge Forest. The composition concerns land, lake and wetland.

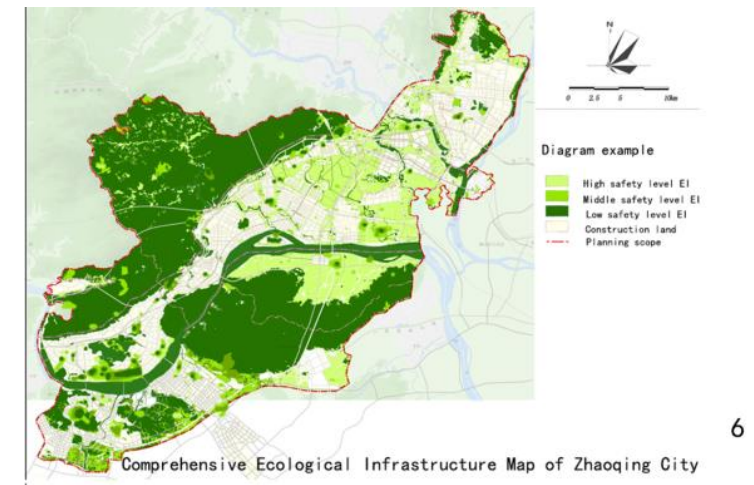

Figure 6. Zhaoqing City comprehensive infrastructure map

Besides, ecological buffer zone accounted for 55.64\%, while development and construction zone and $22.36 \%$, respectively. The ecological buffer zone as the ecological source of the protection wall should be limited development, but the proportion should not be less than $30 \%$;

In addition, the potential ecological corridors are mainly composed of waters, forests and cultivated land. The Xijiang River, the Beijiang River and the lakes and the surrounding cultivated land and forest land together constitute an ecological corridor, which plays a role in connectivity.

Moreover, ecological source areas, ecological corridors and ecological buffers together constitute the ecological security pattern of Zhaoqing City. In short, the ecological pattern of Zhaoqing City has formed a spatial pattern of "one area, two corridors and three cores" relying on natural local conditions.

By superimposing construction land on ecological infrastructures with different safety levels, it can be seen that the ecological conflict space in Zhaoqing City is mainly concentrated along the Beiling Mountains and the Xijiang River Basin and Xinghu Lake. Drawing on the optimal landscape pattern of "segregation between clusters", according to the characteristics of the Zhaoqing area and the actual land use pattern, the "matrix-corridor-plaque" model has become the regional ecological security and the urban and rural non-construction land structure. Inevitably, the "Mountain-ReservoirFarmland-City-Xijiang River" model is a concrete manifestation of the research paradigm of landscape pattern of landscapes. As the ecological bottom line of landscape ecological security pattern, urban and rural non-construction land must protect these bottom lines as ecological infrastructure, in order to realize the basic pattern of Zhaoqing livable city, which has far-reaching strategic significance for scientific planning urban development and expansion.

The study proposes to increase the ecological protection of Zhaoqing ecological source, strengthen the construction of ecological protection measures, find and control the source of ecological safety hidden dangers, and inhibit the development of bad trends. It helps to actively regulate the ecological buffer zone for ecological security 
deterioration in the region, and achieve positive effect on urban ecological security management and monitoring. It is suggested that in the process of building a specific ecological security infrastructure, it is necessary to cooperate with relevant government departments, take into account the development goals of the city, and conduct in-depth research in actual operations.

\section{REFERENCES}

[1] Ahern, J. (1995): Greenways as a planning strategy. - Landscape and Urban Planning 33(1-3): 131-155.

[2] Costanza, R. (2008): Ecosystem services: multiple classification systems are needed. Biological Conservation 141(2): 350-352.

[3] Gan, H. X, Deng, J. H., Zheng, B. (2007): Calculation of inundation range based on GIS. - People's Pearl River 6: 98-100.

[4] Jongman, R. H. G. (1995): Nature conservation planning in Europe: developing ecological networks. - Landscape and Urban Planning 32: 169-183.

[5] Ndubisi, F. (2002): Ecological Planning: A Historical and Comparative Synthesis. Johns Hopkins University Press, Baltimore.

[6] Russell, H, Wang, J, B. (1997): People and the Land through Time: Linking Ecology and History. - Yale University Press, New Haven.

[7] Tony, H., Wang, J. B. (2014): Ecological landscape, water sensitive urban design and green infrastructure. - Chinese Garden 4: 20-24.

[8] Xiao, H. B., Sheng, S, Liu, J. (2017): Study on construction of ecological infrastructure in Foshan City based on landscape ecological security pattern evaluation. - Chinese Garden 33(11): 118-122.

[9] Yu, K. J. (1996): Security patterns and surface model in landscape planning. - Landscape and Urban Planning 36(5): 1-17.

[10] Yu, K. J. (1999): Landscape ecological security pattern of biological protection. - Journal of Ecology 19(1): 8-15.

[11] Yu, K. J, Li, D. H., Liu, H. L. (2005): The "Counter-Planning" Approach. - China Construction Industry Press, Beijing. 